OPEN ACCESS

Edited by:

Dmitri Samovski,

Washington University School

of Medicine in St. Louis, United States

Reviewed by:

Lindsay Brown,

The University of Queensland,

Australia

Jing-Jing Zhou,

The University of Texas MD Anderson

Cancer Center, United States

${ }^{*}$ Correspondence:

Min Shi

sm8344@sina.com

${ }^{\dagger}$ These authors have contributed equally to this work

Specialty section:

This article was submitted to Lipid and Fatty Acid Research, a section of the journal

Frontiers in Physiology

Received: 03 October 2019

Accepted: 13 January 2020

Published: 30 January 2020

Citation:

Cui F. Hu HF, Guo J, Sun J and Shi M (2020) The Effect of Autophagy

on Chronic Intermittent Hypobaric Hypoxia Ameliorating Liver Damage in Metabolic Syndrome Rats. Front. Physiol. 11:13 doi: 10.3389/fphys.2020.00013

\section{The Effect of Autophagy on Chronic Intermittent Hypobaric Hypoxia Ameliorating Liver Damage in Metabolic Syndrome Rats}

\author{
Fang Cui ${ }^{1+}$, Hao Fei Hu${ }^{2 \dagger}$, Jing Guo ${ }^{2}$, Jie Sun ${ }^{2}$ and Min Shi²* \\ 1 Department of Electron Microscope Laboratory Centre, Hebei Medical University, Shijiazhuang, China, ${ }^{2}$ Department \\ of Clinical Laboratory, Second Hospital of Hebei Medical University, Shijiazhuang, China
}

\begin{abstract}
Aim: Our previous study demonstrated that chronic intermittent hypobaric hypoxia $(\mathrm{ClHH})$ can confer hepatic protection by reducing endoplasmic reticulum stress (ERS) in high-fat-high-fructose induced metabolic syndrome (MS) rats. It is known that there is a functional coupling between autophagy and ERS. This study aimed to investigate the effect of $\mathrm{ClHH}$ on autophagy function and adenosine mono-phosphate-activated protein kinase-mammalian target of rapamycin (AMPK $\alpha-m T O R$ ) signaling pathway in hepatic tissue of MS rats.
\end{abstract}

Main Methods: 6-week old male Sprague-Dawley rats were randomly divided into: control $(\mathrm{CON}), \mathrm{ClHH}$ (treated with hypobaric hypoxia simulating 5000-m altitude for 28 days, $6 \mathrm{~h}$ daily), MS (induced by 16-week high fat diet and 10\% fructose water feeding), and $\mathrm{MS}+\mathrm{CHH}$ groups (exposed to $\mathrm{CHH}$ after 16-week MS model). Food and water intakes, body weight, Lee's index, fat coefficient, systolic arterial pressure, blood biochemicals, and histopathology of liver were measured, the expression of phosphorylated (p)-AMPK, p-mTOR, autophagy-related and ERS-related proteins were assayed in hepatic tissue.

Key Findings: The MS rats displayed obesity, hypertension, polydipsia, glucose and lipids metabolism disorders, increased inflammatory cytokine, hepatic tissue morphological and functional damage, and the up-regulated expressions of ERSrelated, autophagy-related proteins and p-mTOR, and the down-regulated expression of $\mathrm{p}$-AMPK $\alpha$. All aforementioned abnormalities in MS rats were ameliorated in $\mathrm{MS}+\mathrm{CHH}$ rats.

Significance: In conclusion $\mathrm{ClHH}$ confers hepatic protection through activating AMPK-mTOR signaling pathway and the autophagy function, thus inhibiting ERS in hepatic tissue.

Keywords: chronic intermittent hypobaric hypoxia, metabolic syndrome, endoplasmic reticulum stress, autophagy, AMPK-mTOR signaling pathway 


\section{INTRODUCTION}

Metabolic syndrome (MS) is a metabolic disorder syndrome characterized by obesity, hyperlipidemia, atherosclerosis, hypertension, diabetes, etc. (Perez-Martinez et al., 2017). Recently, the proportion of obesity patients is rapid increasing worldwidely, and MS has become the first type of disease that endangers human health (Sinaga et al., 2018). As one of the key organ in body metabolism and energy storage, the liver is vulnerable to various stresses. Non-alcoholic fatty liver disease (NAFLD), the main expression of the MS and obesity in liver, promotes liver fibrosis in chronic liver diseases and increases the incidence of cirrhosis and liver cancer (Pagliassotti et al., 2016; Takaki et al., 2014; Zhang et al., 2014). Endoplasmic reticulum stress (ERS) mainly manifests as unfolded or misfolded protein accumulation. Studies have shown that ERS-mediated apoptosis is highly related to liver damage caused by viral hepatitis and NAFLD, which plays a crucial role in the pathogenesis of NAFLD (Lee et al., 2012; Zhang et al., 2012).

Autophagy is an evolutionarily conserved intracellular catabolic process that allows for the degradation and the turnover of damaged proteins and organelles in lysosomes. As one of essential housekeeping mechanisms to resist cell stresses (such as oxidative stress and ERS) and maintain intracellular homeostasis, autophagy is also known as autophagy flux for three major steps: autophagosome formation, fusion with lysosome, and eventual degradation, which is tightly regulated by many proteins encoded by autophagy-related genes (Yasuda-Yamahara et al., 2015). Moderate autophagy can inhibit ERS overactivation, reduce endoplasmic reticulum burden, and exert cytoprotective effects; while defective autophagy aggravates ERS, leads to defects in peripheral tissue insulin signaling pathways, which is associated with various diseases including cancer, neurodegenerative diseases as well as obesity-related cardio-metabolic diseases (De Meyer and Martinet, 2009).

The mammalian target of rapamycin (mTOR), which regulates cell growth and proliferation, maintains cellular energy homeostasis, and inhibits autophagy by inhibiting the formation of lysosomal and autophagy-related protein complexes. Adenosine monophosphate-activated protein kinase (AMPK), an upstream regulatory protein of mTOR, acts both as an upstream energy sensor and as a downstream autophagy activator. AMPK-mTOR signaling pathway is an important signaling regulatory pathway and plays an important role in autophagy.

Chronic intermittent hypobaric hypoxia $(\mathrm{CIHH})$ simulates the plateau environment via low-pressure and low-oxygen conditions. Previous studies have shown that $\mathrm{CIHH}$ have beneficial effects on various tissues of the body (Cui et al., 2018; Shi et al., 2015; Tian et al., 2016; Yuan et al., 2015; Zhou et al., 2013), including improving ischemia/reperfusion or hypoxia/reoxygenation induced tissues damage (Zhou et al., 2013); improving endothelial dysfunction and vascular relaxation in mesenteric arteries (Cui et al., 2018); anti-inflammatory effect by reducing the level of collagen-induced arthritis inflammatory factors (Shi et al., 2015); improving fructose induced liver damage by inhibiting ERS (Yuan et al., 2015). However, the effect and mechanisms of autophagy on $\mathrm{CIHH}$ inhibiting ERS in the liver of rats, is still unclear. In this study, we tested the hypothesis that $\mathrm{CIHH}$ might ameliorate hepatic damage through improving AMPK-mTOR signaling pathway and autophagy, thus inhibiting ERS in high-fat-high-fructose induced MS rats.

\section{MATERIALS AND METHODS}

\section{Fructose Feeding and $\mathrm{ClHH}$ Treatment}

6-week old male Sprague-Dawley rats (body weight: $80 \sim 120 \mathrm{~g}$ ) were provided by the Animal Center of Hebei Medical University. All experiments were carried out in compliance with the Guide for the Care and Use of Laboratory Animals as adopted and promulgated by the U.S. National Institutes of Health, and were reviewed and approved by the Ethics Committee for the Use of Experimental Animals in Hebei Medical University.

6-week old Sprague-Dawley rats (body weight: $80 \sim 120 \mathrm{~g}$ ) were randomly divided into four groups: control group $(\mathrm{CON})$, $\mathrm{CIHH}$ treatment group, metabolic syndrome model group (MS), metabolic syndrome model plus $\mathrm{CIHH}$ treatment group (MS $+\mathrm{CIHH}$ ). $\mathrm{CON}$ rats and $\mathrm{CIHH}$ rats were fed with chow diet $(22 \%$ protein, $4 \%$ fat, and $50 \%$ carbohydrate; nutrient ratio, specific composition per 1,000 g: $99.50 \mathrm{~g}$ water, $216.97 \mathrm{~g}$ protein, $50.38 \mathrm{~g}$ fat, $56.87 \mathrm{~g}$ coarse ash, $24.00 \mathrm{~g}$ fiber, $13.29 \mathrm{~g}$ calcium, $9.17 \mathrm{~g}$ phosphorus) and drinking water. MS rats and MS + CIHH rats were fed with high fat diet $(24 \%$ protein, $12 \%$ fat, and $42 \%$ carbohydrate; nutrient ratio, specific composition: $8 \%$ lard, $2 \%$ soy flour, and $90 \%$ chow diet) and water supplemented with $10 \%(\mathrm{wt} / \mathrm{vol})$ fructose. Sixteen weeks later, $\mathrm{CIHH}$ and $\mathrm{MS}+\mathrm{CIHH}$ rats were exposed to hypobaric hypoxia (simulation of $5000 \mathrm{~m}$ altitude for 28 days in a hypobaric chamber, $6 \mathrm{~h} /$ day, $\mathrm{Po}_{2}=84 \mathrm{~mm} \mathrm{Hg}$ ), as described in our previous studies (Cui et al., 2018). All animals had free access to water and food and were housed in a temperature-controlled room $\left(22 \pm 1^{\circ} \mathrm{C}\right)$ with a $12 \mathrm{~h} / 12 \mathrm{~h}$ light/dark cycle. During the experiment, body weight and systolic arterial pressure (SAP, Panlab model LE5001, Barcelona, Spain) were measured in conscious rats at a fixed time every week. During the 4 weeks of $\mathrm{CIHH}$, the food and water intakes were also measured.

\section{Adipose Assay}

At the end of the experiments, rats were fasted overnight and euthanized with a sodium pentobarbital overdose $(50 \mathrm{mg} / \mathrm{kg}$, intraperitoneal). Body weight and length were measured to calculate the Lee's index [body weight $\times 1,000^{1 / 3} /$ length (Miao et al., 2019). Mesenteric, epididymal, and perirenal fats were collected and weighted to derive the fat coefficient (fat weight/body weight) $\times 100 \%$ ] (Shao et al., 2007).

\section{Blood Biochemical Assay}

Blood samples were collected from the inferior vena cava of rats and centrifuged to get serum (3500 rpm, $10 \mathrm{~min}$ ). Ultraviolet spectrophotometry was performed to determine 
the level of fasting blood glucose according to the principle of hexokinase reaction with automatic biochemical analyzer. Colorimetry was performed to determine the level of cholesterol, triglyceride, high density lipoprotein, low density lipoprotein, alanine transaminase (ALT), and aspartate aminotransferase (AST). Radioimmunoassay was performed to determine the level of insulin and homeostatic model assessment-insulin resistance (HOMA-IR) scores were calculated as fasting blood glucose $\times$ insulin/22.5 (Bonora et al., 2000). Enzymelinked immunosorbent assay (ELISA) was performed to determine the level of interleukin6 (IL6) and tumor necrosis factor- $\alpha(\mathrm{TNF}-\alpha)$.

\section{HE Staining}

Before collecting liver sample, the blood was removed through perfusing with saline $\left(4^{\circ} \mathrm{C}\right)$ in vivo. A small segment of the hepatic tissue was fixed in $4 \%$ paraformaldehyde for $12 \mathrm{~h}$, dehydrated and embedded in paraffin, cut into 4 - $\mu$ m-thick sections, stained with hematoxylin-eosin (HE), and then observed under an optical microscope (BX 50, Olympus Optical, Japan).

\section{Western Blot Analysis}

The protein expression of AMPK $\alpha$, phosphorylated (p)AMPK $\alpha$ (Thr172), mTOR, p-mTOR (ser2448), 78KD glucose-regulated protein (GRP78), C/EBP-homologous protein (CHOP), Beclin-1, microtubule associated protein 1 light chain 3 (LC3), p62 was analyzed by Western blotting (Cui et al., 2018). Briefly, hepatic samples (100 mg) were collected and extracted for protein (centrifuged at $12000 \mathrm{rpm}$, $\left.15 \mathrm{~min}, 4^{\circ} \mathrm{C}\right)$. All samples $(20 \sim 150 \mu \mathrm{g})$ were normalized according to the protein concentrations, separated by $7.5 \sim 15 \%$ SDS-PAGE and transferred to polyvinylidene difluoride (PVDF) membranes. The blots were incubated with primary antibodies overnight at $4^{\circ} \mathrm{C}$ according to the instructions respectively. The reaction was visualized by the chemiluminescence, and the protein contents were normalized to GADPH.

\section{Chemicals}

Fructose was purchased from AMRESCO (Solon, OH, United States). The kit for $\mathrm{HE}$ was purchased from Roche Applied Science (Indianapolis, IN, United States). BCA Protein Assay Kit was obtained from Tiangen Biotech (Beijing, China). Antibody against GRP78 (ab21685) was purchased from Abcam (Cambridge, United Kingdom). Antibody against p-AMPK $\alpha$

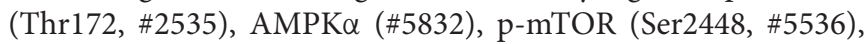
mTOR (\#2983), CHOP (\#2895), Beclin-1 (\#3495), and LC3 (\#2775) were purchased from Cell Signaling Technology (Danvers, MA, United States). Antibody against p62 was purchased from Proteintech Group (Chicago, IL, United States). Antibody against GAPDH was purchased from Antibody Revolution (San Diego, CA, United States). All secondary antibodies were purchased from KPL (Gaithersburg, MD, United States). Enhanced chemiluminescence kit and PVDF membranes were obtained from Millipore Corporation (Billerica,
MA, United States). ELISA kits were purchased from Shanghai Enzyme-linked Biotechnology Co., Ltd. (Shanghai, China).

\section{Statistical Analysis}

All data were expressed as mean \pm standard deviation (mean $\pm \mathrm{SD}$ ). One-way analysis of variance (one-way ANOVA) was used to compare multiple sets of data, and multiple comparisons between the two groups were performed using the Student-Newman-Keuls test (SNK test). $P<0.05$ was considered to be statistically significant.

\section{RESULTS}

\section{Effects of CIHH on Body Weight, SAP, Food Intakes, Water Intakes, Lee's Index and Fat Coefficient}

At the beginning of the experiment, body weight and SAP showed no statistical difference among four groups $(P>0.05)$. During the 16-week development of MS model, the body weights and SAP of the rats fed with the high-fat, high-fructose diet were heavier than those with the chow diet and drinking water $(P<0.05$; Figures 1A,B), and in terms of the water intakes, the former were more than the latter $(P<0.01$, Figure 1D), even though the food intakes were not different $(P>0.05$, Figure 1C). During 4 weeks of $\mathrm{CIHH}$ treatment, there were not different in the food and water intakes of four groups rats, compared with before $\mathrm{CIHH}$ treatment. After 4 weeks of CIHH treatment, body weight, Lee's index, fat coefficient and SAP were decreased in MS $+\mathrm{CIHH}$ rats compared with MS rats $(P<0.05$, Figures $\mathbf{1 A}, \mathbf{B}$, and Table $\mathbf{1})$, while no significant difference between $\mathrm{CIHH}$ and $\mathrm{CON}$ rats $(P>0.05$, Figures 1A,B, and Table 1). These data showed that $\mathrm{CIHH}$ could effectively antagonize polydipsia, obesity and hypertension in MS rats.

\section{Effect of ClHH on Blood Biochemical Parameters Effect of CIHH on Glucose and Lipid Metabolism}

Fasting blood glucose, insulin, HOMA-IR scores, cholesterol, triglyceride and low-density lipoprotein were significantly increased in MS rats compared with CON rats, and decreased in $\mathrm{MS}+\mathrm{CIHH}$ rats compared with $\mathrm{MS}$ rats $(P<0.05, P<0.01$, Figures $2 \mathbf{A}-\mathbf{F})$. High-density lipoprotein was significantly decreased in MS rats compared with $\mathrm{CON}$ rats, and increased in $\mathrm{MS}+\mathrm{CIHH}$ rats compared with MS rats $(P<0.05$, Figure 2G). The results indicated that $\mathrm{CIHH}$ treatment could improve glucose and lipid metabolism disorders and insulin resistance in MS rats.

\section{Effect of CIHH on the Level of Inflammatory Factor}

Serum IL6 and TNF- $\alpha$ level was increased in MS rats compared with $\mathrm{CON}$ rats $(P<0.01)$ and decreased in $\mathrm{MS}+\mathrm{CIHH}$ rats compared with MS rats $(P<0.01$; Figures $2 \mathrm{H}, \mathbf{I})$. The results indicated that $\mathrm{CIHH}$ treatment could decrease inflammatory response in MS rats. 

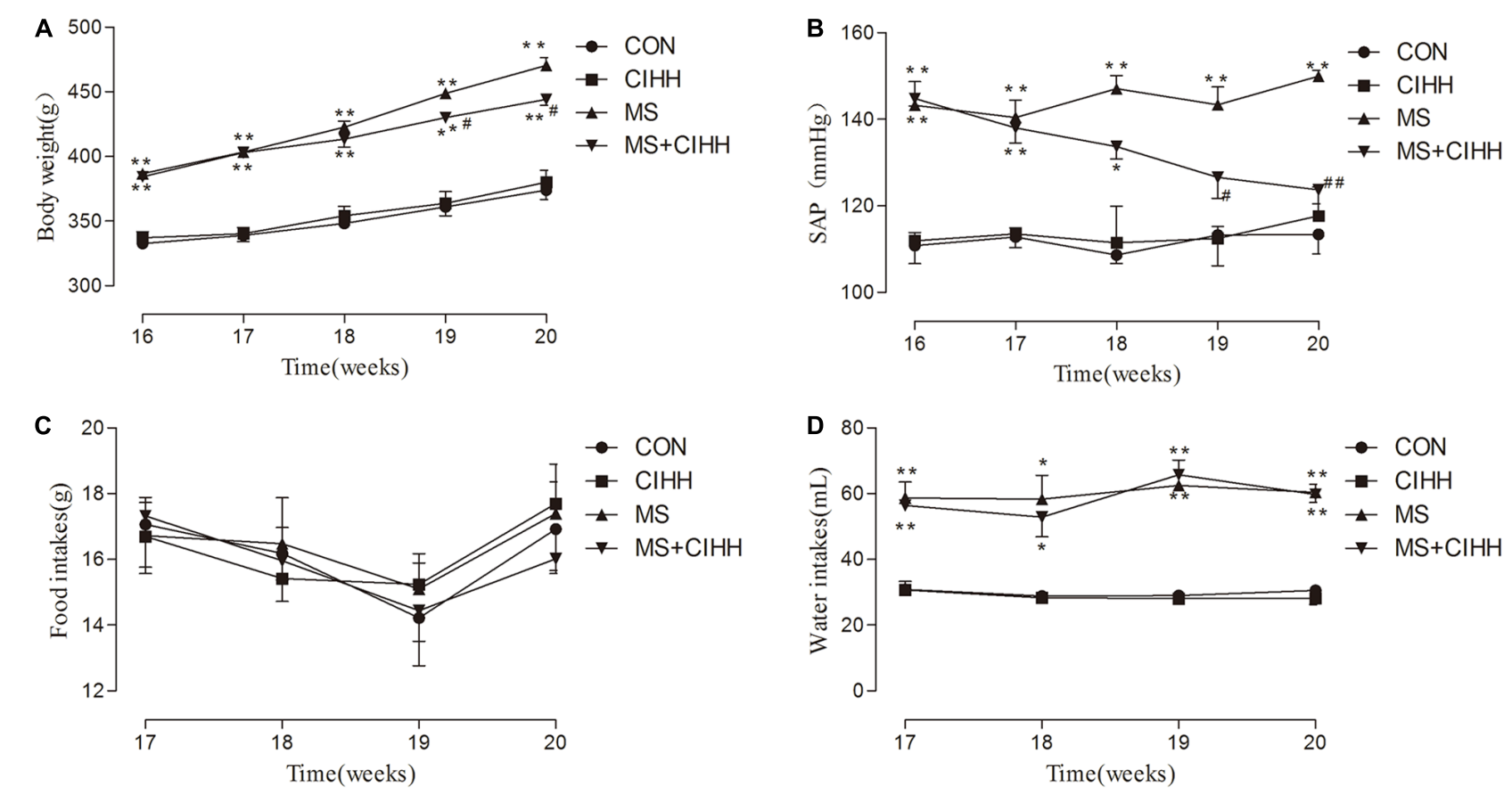

FIGURE 1 | The effect of $\mathrm{ClHH}$ on body weight, systolic arterial pressure (SAP), food and water intakes. (A) The effect of ClHH on body weight. (B) The effect of $\mathrm{CIHH}$ on SAP. (C) The effect of $\mathrm{ClHH}$ on food intakes. (D) The effect of $\mathrm{CHHH}$ on water intakes. CON: control group, $\mathrm{ClHH}$ : $\mathrm{ClHH}$ group, $\mathrm{MS}$ : metabolic syndrome group, $\mathrm{MS}+\mathrm{CHH}$ : MS $+\mathrm{CHH}$ group. All data were expressed as mean $\pm \mathrm{SD} ; n=5-6$ for each group. ${ }^{\star} P<0.05{ }^{\star \star} P<0.01$ vs. CON, $\# P<0.05 \# \# P<0.01$ vs. MS.

\section{Effect of CIHH on Hepatic Tissue Effect of CIHH on Liver Morphology}

As shown in Figure 3, HE staining revealed that the hepatic tissues of MS rats had obvious fatty degeneration and some cord-like changes compared with those of CON rats, while the morphology of hepatic tissues in $\mathrm{MS}+\mathrm{CIHH}$ rats was significantly improved compared with MS rats. The results indicated that $\mathrm{CIHH}$ treatment could improve hepatic tissues morphological damage in MS rats.

\section{Effect of ClHH on Liver Function}

Compared with CON rats, the level of serum ALT and AST in MS rats was significantly increased $(P<0.05$, Figure 4). Compared with MS rats, the level of serum ALT and AST in MS + CIHH rats was significantly lower $(P<0.05$, Figure 4$)$. The results indicated that $\mathrm{CIHH}$ treatment could improve hepatic tissues functional damage in MS rats.

TABLE 1 | Effect of $\mathrm{CHHH}$ on Lee's index and fat coefficient.

\begin{tabular}{lcccc}
\hline & CON & CIHH & MS & MS + CIHH \\
\hline Lee's index & $2.96 \pm 0.10$ & $2.92 \pm 0.10$ & $3.18 \pm 0.04^{\star}$ & $2.93 \pm 0.04^{\#}$ \\
Fat coefficient & $1.67 \pm 0.18$ & $1.64 \pm 0.19$ & $4.30 \pm 1.04^{\star}$ & $2.34 \pm 0.85^{\#}$ \\
\hline
\end{tabular}

Lee's index $=$ body weight $\times 1,0001 / 3 /$ length. Fat coefficient $=$ fat weight (Mesenteric, epididymal, and perirenal fats)/body weight) $\times 100 \%$. CON: control group, ClHH: ClHH group, MS: metabolic syndrome group, $\mathrm{MS}+\mathrm{CHH}$ : $M S+C l H H$ group, All data are expressed as mean $\pm S D, n=6$ for each group, ${ }^{*} P<0.05$ vs. CON, \#P $<0.05$ vs. MS.

\section{Effect of ClHH on Protein Expression Effect of CIHH on Expression of ERS-Related Proteins}

Compared with CON rats, the expression of ERS-related protein, GRP78 and CHOP, in liver tissues was significantly increased in MS rats $(P<0.05$, Figure 5 and Supplementary Figure S1). The protein expression of GRP78 and CHOP in liver tissues was significantly decreased in $\mathrm{MS}+\mathrm{CIHH}$ rats compared with MS rats $(P<0.05$, Figure 5). The results indicated that the expression of ERS-related proteins was up-regulated in MS rats, and $\mathrm{CIHH}$ ameliorated the disturbance of ERS-related proteins in MS rats.

\section{Effect of ClHH on the Expression of Autophagy-Related Proteins}

Compared with CON rats, the expression of Beclin-1, LC3-II/I and p62 in liver tissues was significantly increased in MS rats $(P<0.05$, Figure 6 and Supplementary Figure S2). Compared with MS rats, the expression of Beclin-1, LC3-II/I and p62 in liver tissues was significantly decreased in $\mathrm{MS}+\mathrm{CIHH}$ rats $(P<0.05$, Figure 6). The results indicated that the expression of autophagy-related proteins was up-regulated in MS rats, but down-regulated in $\mathrm{CIHH}$ rats.

\section{Effect of $\mathrm{CIHH}$ on the Expression of p-AMPK $\alpha$ and p-mTOR}

Because autophagy is a dynamic process, the increased protein expression represents completely converse autophagy 

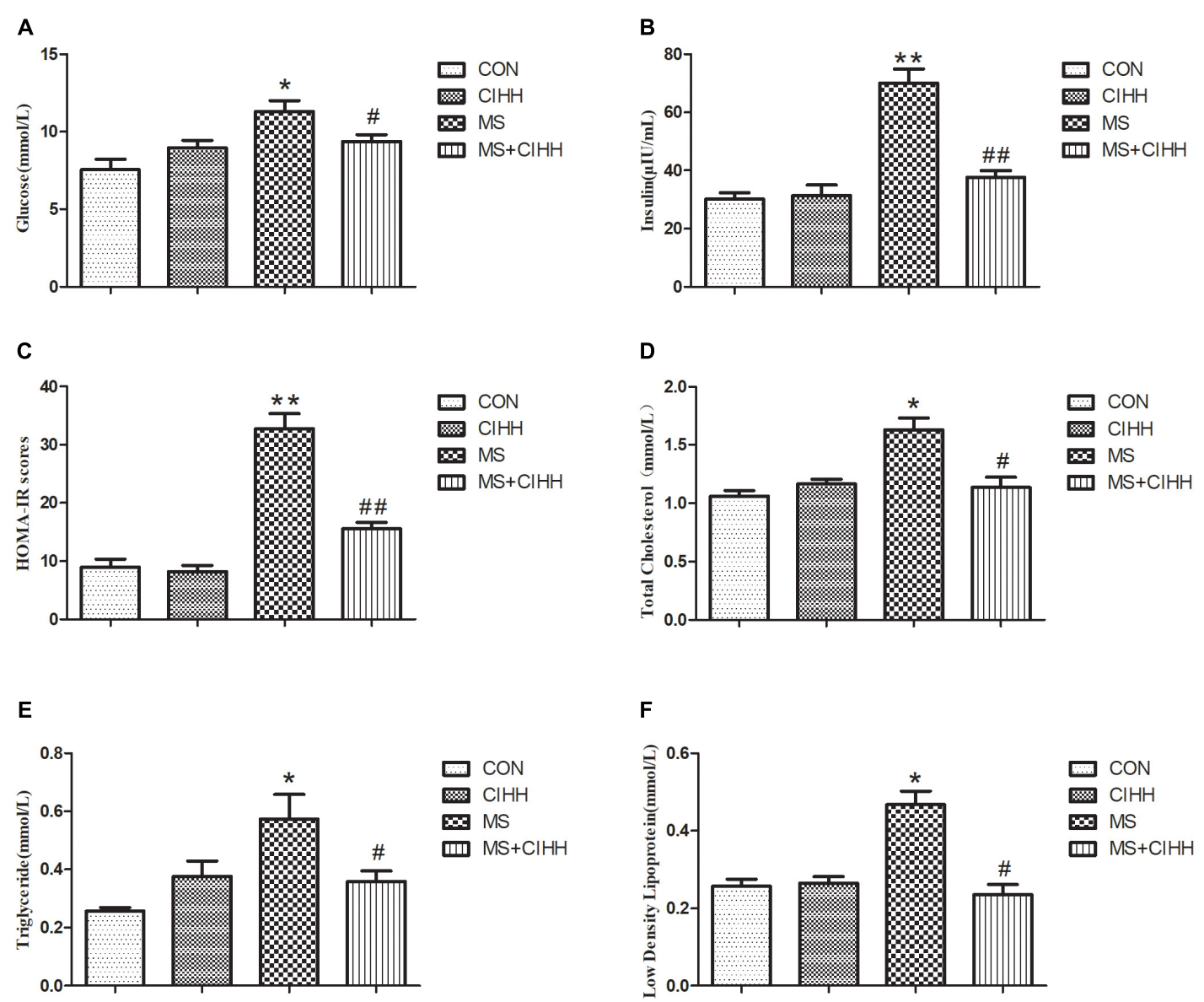

G

H
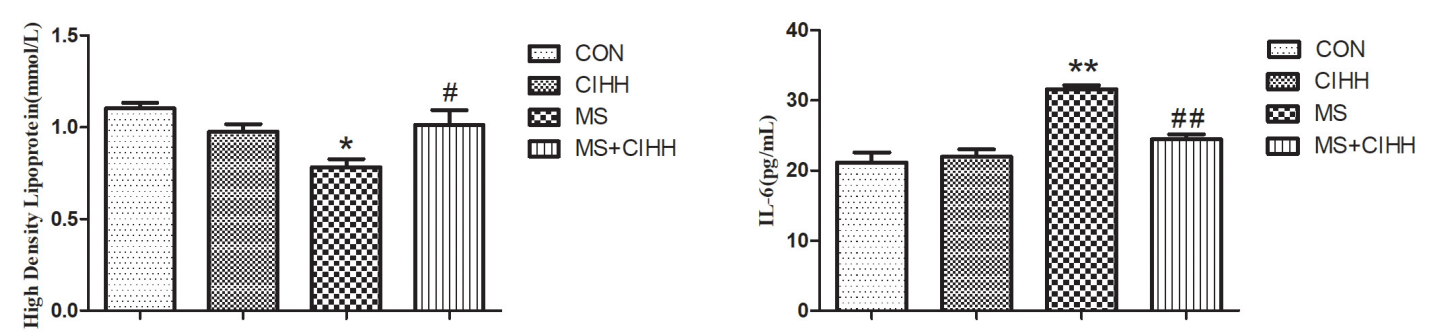

I

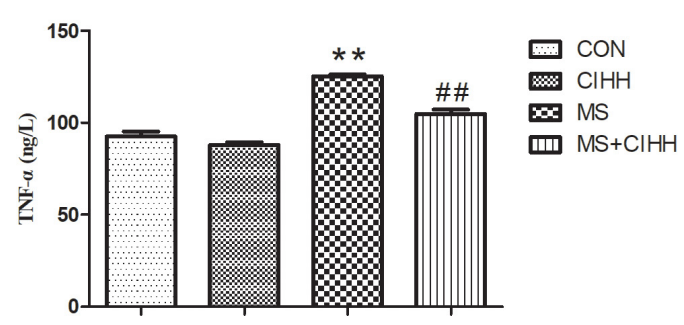

FIGURE 2 | The effect of $\mathrm{ClHH}$ on blood biochemical parameters. (A) The effect of $\mathrm{ClHH}$ on glucose. (B) The effect of $\mathrm{ClHH}$ on insulin. (C) The effect of $\mathrm{ClHH}$ on homeostatic model assessment-insulin resistance (HOMA-IR) scores. (D) The effect of $\mathrm{ClHH}$ on total cholesterol. (E) The effect of ClHH on triglyceride. (F) The effect of $\mathrm{CIHH}$ on low density lipoprotein. (G) The effect of $\mathrm{CIHH}$ on high density lipoprotein. (H) The effect of $\mathrm{CIHH}$ on interleukin 6 (IL6). (I) The effect of CIHH on tumor necrosis factor- $\alpha$ (TNF- $\alpha$ ). CON: control group, $\mathrm{ClHH}$ : ClHH group, MS: Metabolic syndrome group, $\mathrm{MS}+\mathrm{ClHH}$ : MS $+\mathrm{ClHH}$ group. All data were expressed as mean $\pm \mathrm{SD} ; n=6$ for each group. ${ }^{*} P<0.05{ }^{* \star} P<0.01$ vs. CON, $\# P<0.05 \# \# P<0.01$ vs. MS. 


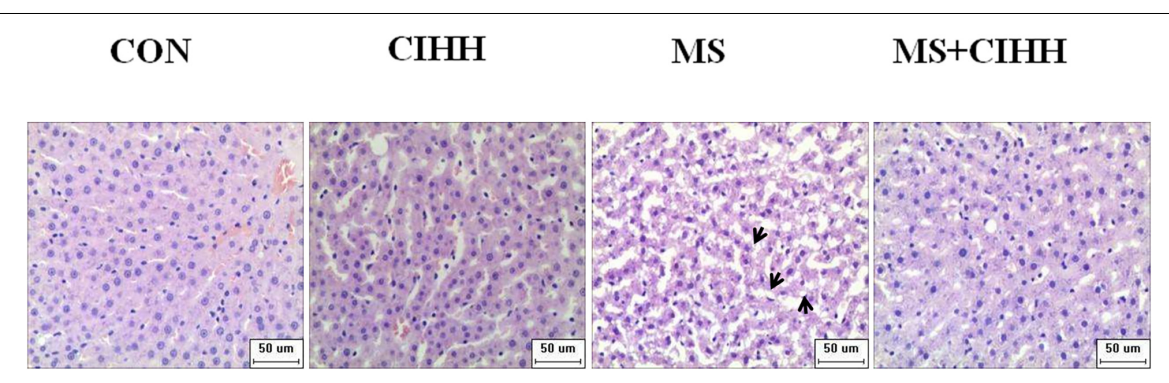

FIGURE 3 | Effect of $\mathrm{CIHH}$ on pathologic morphology of liver. HE staining of liver $(\times 400)$, black arrows $(\uparrow)$ represent fatty degeneration and cord-like changes. $n=4$ for each group.
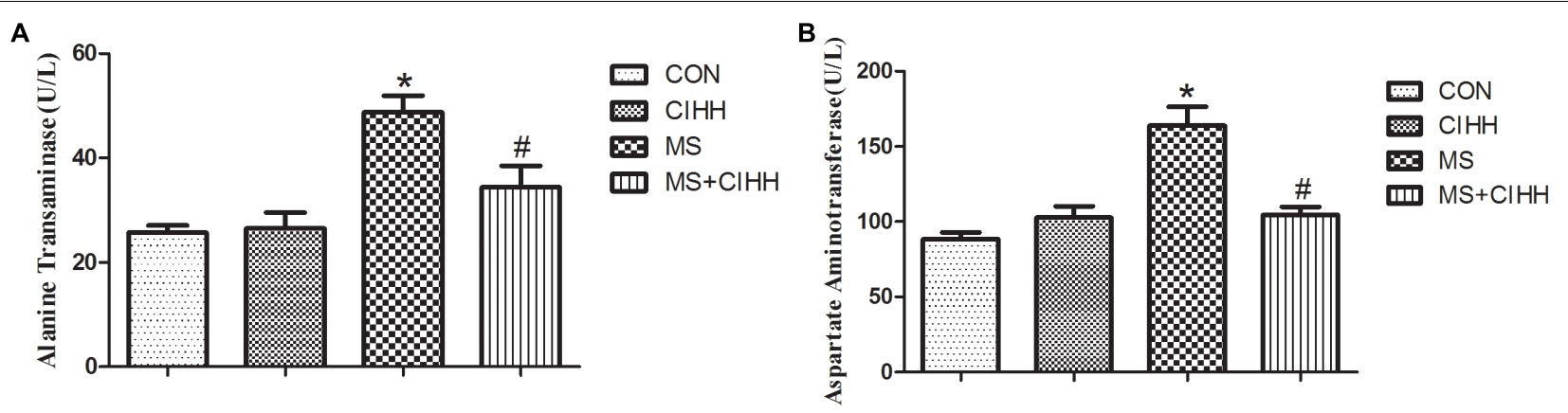

FIGURE 4 | Effect of $\mathrm{ClHH}$ on the level of alanine transaminase (A) and aspartate aminotransferase (B). CON: control group, $\mathrm{CIHH}$ : $\mathrm{ClHH}$ group, MS: metabolic syndrome group, $\mathrm{MS}+\mathrm{ClHH}$ : MS $+\mathrm{ClHH}$ group. All data were expressed as mean $\pm \mathrm{SD} ; n=6$ for each group. ${ }^{*} P<0.05$ vs. CON, $\# P<0.05$ vs. MS.
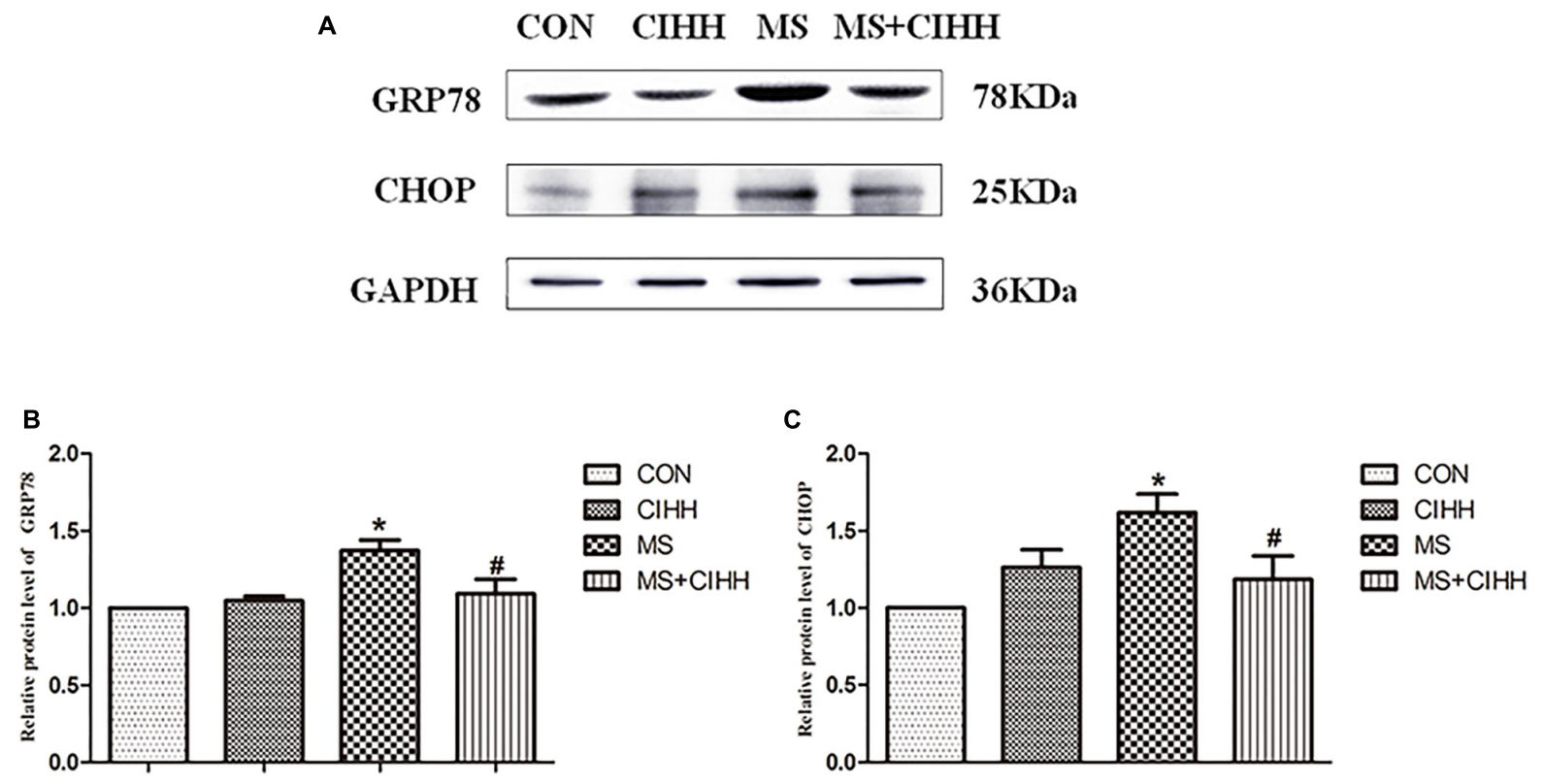

FIGURE 5 | Effect of $\mathrm{CIHH}$ on the expression of ERS-related proteins in rat livers. (A) Original recording of Western blotting. (B) Quantification of protein levels of GRP78. (C) Quantification of protein levels of $\mathrm{CHOP}$. CON: control group, $\mathrm{ClHH}$ : $\mathrm{ClHH}$ group, $\mathrm{MS}$ : metabolic syndrome group, $\mathrm{MS}+\mathrm{ClHH}$ : $\mathrm{MS}+\mathrm{ClHH}$ group. All data were expressed as mean $\pm \mathrm{SD} ; n=4$ for each group. ${ }^{*} P<0.05$ vs. CON, $\# P<0.05$ vs. MS.

function: improvement or inhibition. Therefore, we further observed the effect of $\mathrm{CIHH}$ on one signaling pathway of inducing autophagy - AMPK-mTOR signaling pathway.
Compared with CON rats, the level of p-AMPK $\alpha$ (Thr172) was significantly down-regulated in MS rats $(P<0.05$, Figure 7 and Supplementary Figure S3). Compared with MS rats, the 
A

\section{CON CIHH MS MS+CIHH}
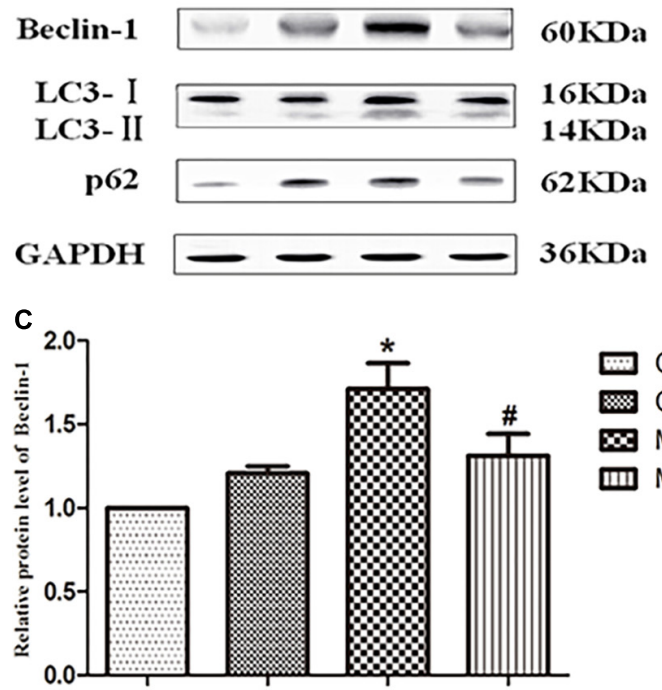

B

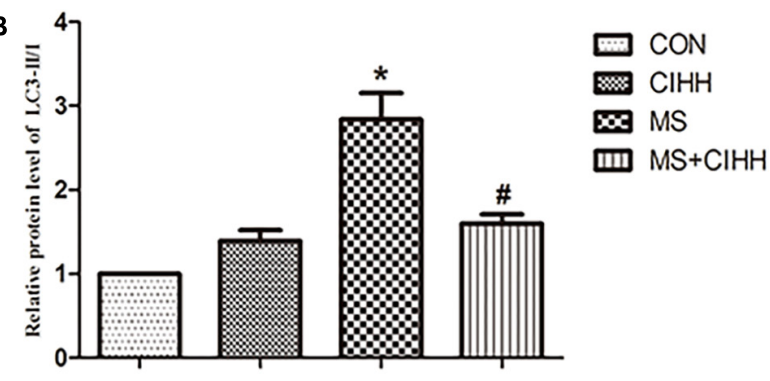

D

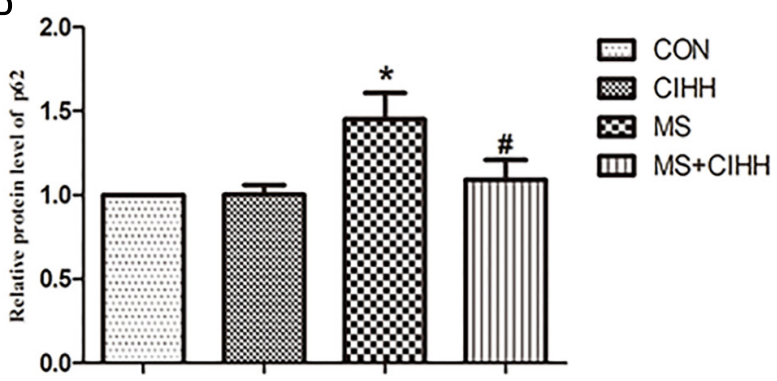

FIGURE 6 | Effect of $\mathrm{CIHH}$ on the expression of autophagy-related proteins in rat livers. (A) Original recording of Western blotting. (B) Quantification of protein levels of LC3-II/I. (C) Quantification of protein levels of Beclin-1. (D) Quantification of protein levels of p62. CON: control group, ClHH: ClHH group, MS: metabolic syndrome group, $\mathrm{MS}+\mathrm{ClHH}$ : MS $+\mathrm{ClHH}$ group. All data were expressed as mean $\pm \mathrm{SD} ; n=4$ for each group. ${ }^{\star} P<0.05$ vs. CON, \#P<0.05 vs. MS.

A

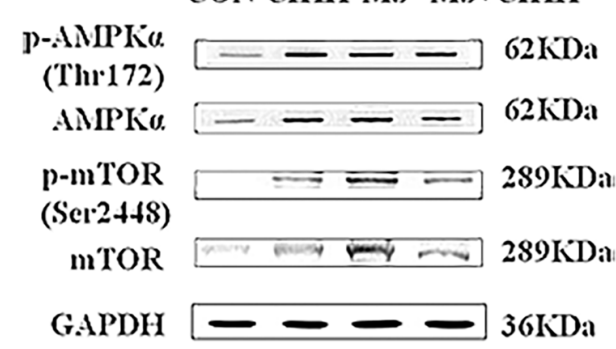

B

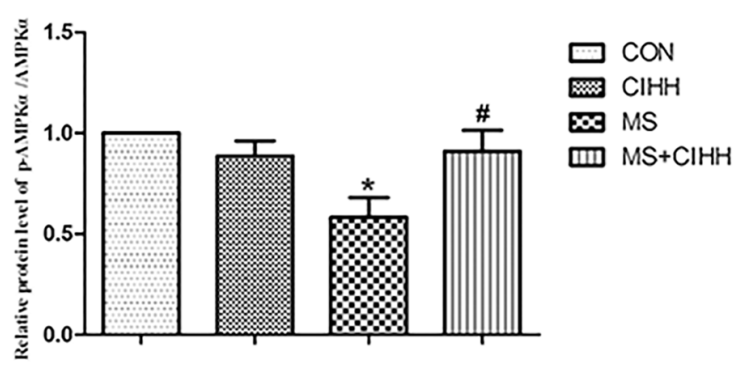

C

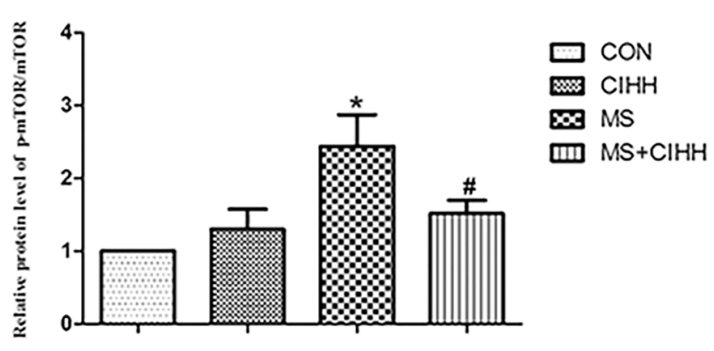

FIGURE 7 | Effect of ClHH on the phosphorylation of AMPK $\alpha$ (Thr172) and mTOR (Ser2448) in rat livers. (A) Original recording of Western blotting. (B) Quantification of protein levels of p-AMPKa. (C) Quantification of protein levels of p-mTOR. CON: control group, $\mathrm{ClHH}$ : ClHH group, MS: metabolic syndrome group, $\mathrm{MS}+\mathrm{ClHH}$ : $\mathrm{MS}+\mathrm{ClHH}$ group. All data were expressed as mean $\pm \mathrm{SD} ; n=4$ for each group. ${ }^{*} P<0.05$ vs. CON, $\# P<0.05$ vs. MS.

level of $\mathrm{p}$-AMPK $\alpha$ (Thr172) was significantly up-regulated in $\mathrm{MS}+\mathrm{CIHH}$ rats $(P<0.05$, Figure 7$)$.

Compared with CON rats, the level of p-mTOR (Ser2448) was significantly up-regulated in MS rats $(P<0.05$, Figure 7$)$.
Compared with MS rats, the level of p-mTOR (Ser2448) was significantly down-regulated in $\mathrm{MS}+\mathrm{CIHH}$ rats $(P<0.05$, Figure 7). The results indicated that the expression of $\mathrm{p}-\mathrm{AMPK} \alpha$ was down-regulated and the expression of $\mathrm{p}$-mTOR was 
up-regulated in MS rats, and CIHH actived the AMPK-mTOR signaling pathway in MS rats.

\section{DISCUSSION}

The beneficial effect of $\mathrm{CIHH}$ on the impaired hepatocyte was investigated in high-fat-high-fructose induced MS rats. Compared with CON rats, MS rats showed polydipsia, obesity, hypertension, glucose and lipids metabolism disorders, increased inflammatory cytokine, liver tissue morphological and functional damage, and increased expression of ERS-related and autophagyrelated proteins. The phosphorylation level of AMPK $\alpha$ was down-regulated while mTOR was up-regulated. CIHH treatment alleviated all changes in MS rats effectively, which suggested $\mathrm{CIHH}$ ameliorated hepatic damage through activating AMPKmTOR signaling pathway, up-regulating autophagy, thus inhibiting ERS in MS rats.

MS is mainly characterized by obesity, hypertension, dyslipidemia, diabetes and insulin resistance. NAFLD is one of the main components and manifestation of MS which is used as the risk evaluation of type 2 diabetes and MS by the American Association of Clinical Endocrinologists (Pedro-Botet et al., 2018; Wang et al., 2006), because it further exacerbates other types of liver disease, such as viral hepatitis and alcoholic liver disease, as well as affects its therapeutic effect (Corte et al., 2015). $\mathrm{CIHH}$ simulates the plateau environment of low-pressure and hypoxic conditions, and the experimental animals are placed in this environment discontinuously. Our previous studies have shown that $\mathrm{CIHH}$ can improve rat liver injury induced by fructose feeding by inhibiting ERS (Yuan et al., 2015). In this study, MS rats fed with 16 weeks high-fat-high-fructose diet had pathological manifestations, liver damage, and can be significantly improved after 28 days of CIHH treatment.

The endoplasmic reticulum is an organelle in the cell which main function is to synthesize, modify, and fold proteins, store calcium ions, and regulate intracellular calcium ion balance. ERS is induced to degradate the accumulation of the wrong protein in the lumen of the endoplasmic reticulum for the maintenance of cell normal function. It is the main causes of NAFLD, that insulin resistance, oxidative stress, obesity and dyslipidemia, cause inflammation and infiltration of simple fatty liver and hepatocytes, and eventually lead to liver fibrosis and cirrhosis (Sangouni and Ghavamzadeh, 2019). Nakatani (Nakatani et al., 2005) found that the development of insulin resistance was closely related to ERS which promote the aggravation of insulin resistance and the insulin resistance of extrahepatic tissues. At the same time, hepatocyte injury is closely related to ERSmediated apoptosis. In patients with alcoholic fatty liver and steatohepatitis, there is obvious hepatocyte apoptosis, and the degree of apoptosis is related to the severity of the disease and liver fibrosis. Thus, studies have shown that patients with diabetes, high-fat diet, and insulin resistance are more likely to develop ERS than normal individuals. ERS may be an important link in the process of liver injury (Nakatani et al., 2006).

GRP78 is a molecular chaperone of the endoplasmic reticulum (Su et al., 2011; Zhao et al., 2014), dissociated from the three stressor proteins on the endoplasmic reticulum membrane (Nakatani et al., 2005) to promote proper folding, assembly and transport of proteins, modification during the activation of ERS. When ERS lasts for a longer time or is more severe, it not only will be compensated by up-regulated expression of GRP78, but also induces the apoptotic pathway and apoptosis-related molecules, such as CHOP, caspase-12, JNK, etc. Therefore, GRP78 and CHOP are the hallmark proteins of ERS (Su et al., 2011; Zhao et al., 2014). Compared with normal-fed KKAy mice and control C57BL/6J mice, the mRNA levels of ERS marker genes GRP78 and CHOP were significantly elevated in white adipose tissue of high-fat diet mice. In the study we found that the up-regulation of GRP78 and CHOP was attenuated by CIHH. It indicated that $\mathrm{CIHH}$ may protect against hepatic damage by ameliorating ERS in hepatic tissue of MS rats.

ERS and autophagy interact and are closely related. ERS induces autophagy to degradate the excessive accumulation of unfolded or misfolded proteins and restore the endoplasmic reticulum homeostasis (Chandrahas et al., 2018; Ogata et al., 2006), which is called as endoplasmic reticulum actived autophagy (ERAA); on the other hand, autophagy can also affect ERS. For example, inhibition of autophagy with chloroquine can aggravate ERS and increase cell death. Autophagy participates in the degradation of lipids and lipid metabolism in hepatocyte, maintains fat homeostasis, and is closely related to the development of fatty liver and insulin resistance. Studies have shown that the up-regulation of autophagy promoted liver fat removal, while the down-regulation lead to the accumulation of lipids. In high-fat diet, the accumulation of lipids in hepatocytes will down-regulate autophagy and further aggravate the accumulation of lipids in hepatocyte, thus form a vicious circle of liver damage and the development of steatohepatitis (Seth et al., 2011). Autophagy can provide survival protection for liver cancer cells in the ERS state, and has no significant effect on normal liver cells. In the study we found that the expression of autophagy-related proteins LC3-II/I, Beclin-1 and p62 was increased in MS rats, and was decreased by $\mathrm{CIHH}$ treatment. Because autophagy is a dynamic process, the increased protein expression represents two different states of autophagy: increased autophagosome synthesis indicates an increase in autophagy function; and blocked degradation indicates a decrease in the autophagy function. Therefore, we further observed the effect of $\mathrm{CIHH}$ on one signaling pathway of regulating autophagy AMPK-mTOR signaling pathway.

The AMPK-mTOR signaling pathway plays an important role in the regulation of autophagy. AMPK, an energy receptor, is a key regulatory site in endocrine diseases such as obesity and diabetes, and activates autophagy by inhibiting mTOR activity. Under normal conditions, AMPK remains dephosphorylated and inactive state; when energy is shortage, such as obesity and MS, AMPK is phosphorylated at the site of Thr172 and actived, which directly phosphorylates the Thr2446 site of mTOR, inhibits Akt-mediated phosphorylation of Ser2448, indirectly inhibits mTOR activity, thus activates autophagy. Studies have shown that high-fat, high-fructose-induced obese mice and rats have abnormal AMPK-mTOR signaling pathways, and vascular endothelial AMPK phosphorylation levels are significantly 
reduced, while mTOR phosphorylation levels are significantly increased (Ma et al., 2010). In the study we found that $\mathrm{CIHH}$ could increase the down-regulated phosphorylation level of $\operatorname{AMPK} \alpha$, and decrease the up-regulated phosphorylation level of mTOR, which suggested that CIHH could activate AMPK-mTOR signaling pathway, then up-regulate the autophagy function in hepatocyte tissue of MS rats. In the light of increased autophagyrelated protein expression in MS rats, we speculated that the accumulation of autophagy-related proteins may be caused by blocked autophagy degradation and defective autophagy function, and it warrants further investigation in future studies.

$\mathrm{CIHH}$ as a training or treatment method for sports and cardiovascular diseases has been reported in former Soviet Union decades ago, and has been proved having lots of beneficial action such as losing of body mass and improvement of metabolic abnormality (Kayser and Verges, 2013). With optimal level and time, $\mathrm{CIHH}$ is promising to become a potential therapy for prevention and treatment of metabolic and cardiovascular dysfunctions in obese and MS patients.

\section{CONCLUSION}

In conclusion, our study demonstrates for the first time that $\mathrm{CIHH}$ confers hepatic protection in high-fat-high-fructose induced MS rats, which might be related to the activation of AMPK-mTOR signaling pathway and the autophagy function, thus the inhibition of ERS.

\section{DATA AVAILABILITY STATEMENT}

All datasets generated for this study are included in the article/Supplementary Material.

\section{REFERENCES}

Bonora, E., Targher, G., Alberiche, M., Bonadonna, R. C., Saggiani, F., and Zenere, M. B. (2000). Homeostasis model assessment closely mirrors the glucose clamp technique in the assessment of insulin sensitivity: studies in subjects with various degrees of glucose tolerance and insulin sensitivity. Diabetes Care 23, 57-63. doi: 10.2337/diacare.23.1.57

Chandrahas, V. K., Han, J., and Kaufman, R. J. (2018). Coordinating organismal metabolism during protein misfolding in the er through the unfolded protein response. Curr. Top. Microbiol. Immunol. 414, 103-130. doi: 10.1007/82_2017_ 41

Corte, C. D., Ferrari, F., Villani, A., and Nobili, V. (2015). Epidemiology and natural history of NAFLD. J. Med. Biochem. 34, 13-17. doi: 10.2478/jomb-20140049

Cui, F., Guan, Y., Guo, J., Tian, Y. M., Hu, H. F., Zhang, X. J., et al. (2018). Chronic intermittent hypobaric hypoxia protects vascular endothelium by ameliorating autophagy in metabolic syndrome rats. Life Sci. 205, 145-154. doi: 10.1016/j.lfs. 2018.05.008

De Meyer, G. R., and Martinet, W. (2009). Autophagy in the cardiovascular system. Biochim. Biophys. Acta 1793, 1485-1495. doi: 10.1016/j.bbamcr.2008.12.011

Kayser, B., and Verges, S. (2013). Hypoxia, energy balance and obesity: from pathophysiological mechanisms to new treatment strategies. Obes. Rev. 14, 579-592. doi: 10.1111/obr.12034

Lee, J. S., Zheng, Z., Mendez, R., Ha, S. W., Xie, Y., and Zhang, K. (2012). Pharmacologic ER stress induces non-alcoholic steatohepatitis

\section{ETHICS STATEMENT}

The animal study was reviewed and approved by the Ethics Committee for the Use of Experimental Animals in Hebei Medical University.

\section{AUTHOR CONTRIBUTIONS}

FC, $\mathrm{HH}$, and JG performed the experiments and drafted the manuscript. JS analyzed the data. MS was responsible for conception and design of the research, revised, and approved the final manuscript.

\section{FUNDING}

This study was supported by the Hebei Province Higher Education Science and Technology Research Project (No. QN2019014).

\section{ACKNOWLEDGMENTS}

We thank Yi Zhang Ph.D. (Department of Physiology, Hebei Medical University, Shijiazhuang, China) for editing the English text of revised manuscript.

\section{SUPPLEMENTARY MATERIAL}

The Supplementary Material for this article can be found online at: https://www.frontiersin.org/articles/10.3389/fphys. 2020.00013/full\#supplementary-material

in an animal model. Toxicol. lett. 211, 29-38. doi: 10.1016/j.toxlet.2012. 02.017

Ma, L., Ma, S., He, H., Yang, D., Chen, X., Luo, Z., et al. (2010). Perivascular fat-mediated vascular dysfunction and remodeling through the AMPK/mTOR pathway in high-fat diet-induced obese rats. Hypertens Res. 33, 446-453. doi: 10.1038/hr.2010.11

Miao, Y. F., Kang, H. X., Li, J., Zhang, Y. M., Ren, H. Y., Zhu, L., et al. (2019). Effect of Sheng-jiang powder on multiple-organ inflammatory injury in acute pancreatitis in rats fed a high-fat diet. World J. Gastroenterol. 25, 683-695. doi: 10.3748/wjg.v25.i6.683

Nakatani, Y., Kaneto, H., Hatazaki, M., Yoshiuchi, K., Kawamori, D., Sakamoto, K., et al. (2006). Increased stress protein ORP150 autoantibody production in type 1 diabetic patients. Diabet Med. 23, 216-219. doi: 10.1111/j.1464-5491.2005. 01766.X

Nakatani, Y., Kaneto, H., Kawamori, D., Yoshiuchi, K., Hatazaki, M., Matsuoka, T. A., et al. (2005). Involvement of endoplasmic reticulum stress in insulin resistance and diabetes. J. Biol. Chem. 280, 847-851. doi: 10.1074/jbc. M411860200

Ogata, M., Hino, S., Saito, A., Morikawa, K., Kondo, S., Kanemoto, S., et al. (2006). Autophagy is activated for cell survival after endoplasmic reticulum stress. Mol. Cell. Biol. 26, 9220-9231. doi: 10.1128/mcb.01453-06

Pagliassotti, M. J., Kim, P. Y., Estrada, A. L., Stewart, C. M., and Gentile, C. L. (2016). Endoplasmic reticulum stress in obesity and obesity-related disorders: an expanded view. Metab 65, 1238-1246. doi: 10.1016/j.metabol.2016. 05.002 
Pedro-Botet, J., Ascaso, J. F., Barrios, V., De la Sierra, A., Escalada, J., Millan, J., et al. (2018). COSMIC project: consensus on the objectives of the metabolic syndrome in clinic. Diabetes Metab. Syndr. Obes. 11, 683-697. doi: 10.2147/ dmso.s 165740

Perez-Martinez, P., Mikhailidis, D. P., Athyros, V. G., Bullo, M., Couture, P., Covas, M. I., et al. (2017). Lifestyle recommendations for the prevention and management of metabolic syndrome: an international panel recommendation. Nutr. Rev. 75, 307-326. doi: 10.1093/nutrit/nux014

Sangouni, A. A., and Ghavamzadeh, S. (2019). A review of synbiotic efficacy in nonalcoholic fatty liver disease as a therapeutic approach. Diabetes Metab. Syndr. 13, 2917-2922. doi: 10.1016/j.dsx.2019.07.063

Seth, D., Haber, P. S., Syn, W. K., Diehl, A. M., and Day, C. P. (2011). Pathogenesis of alcohol-induced liver disease: classical concepts and recent advances. J. Gastroenterol. Hepatol. 26, 1089-1105. doi: 10.1111/j.1440-1746. 2011.06756.x

Shao, H., Reed, D. R., and Tordoff, M. G. (2007). Genetic loci affecting body weight and fatness in a C57BL/6J x PWK/PhJ mouse intercross. Mamm Genome 18, 839-851. doi: 10.1007/s00335-007-9069-6

Shi, M., Cui, F., Liu, A. J., Ma, H. J., Cheng, M., Song, S. X., et al. (2015). The protective effects of chronic intermittent hypobaric hypoxia pretreatment against collagen-induced arthritis in rats. J. Inflamm. 12:23. doi: 10.1186/ s12950-015-0068-1

Sinaga, M., Worku, M., Yemane, T., Tegene, E., Wakayo, T., Girma, T., et al. (2018). Optimal cut-off for obesity and markers of metabolic syndrome for Ethiopian adults. Nutr. J. 17:109. doi: 10.1186/s12937-0180416-0

Su, Y. C., Wu, J. L., and Hong, J. R. (2011). Betanodavirus up-regulates chaperone GRP78 via ER stress: roles of GRP78 in viral replication and host mitochondriamediated cell death. Apoptosis 16, 272-287. doi: 10.1007/s10495-0100565-x

Takaki, A., Kawai, D., and Yamamoto, K. (2014). Molecular mechanisms and new treatment strategies for non-alcoholic steatohepatitis (NASH). Int. J. Mol. Sci. 15, 7352-7379. doi: 10.3390/ijms15057352

Tian, Y. M., Guan, Y., Li, N., Ma, H. J., Zhang, L., Wang, S., et al. (2016). Chronic intermittent hypobaric hypoxia ameliorates diabetic nephropathy through enhancing HIF1 signaling in rats. Diabetes Res. Clin. Pract. 118, 90-97. doi: 10.1016/j.diabres.2016.06.021
Wang, D., Wei, Y., and Pagliassotti, M. J. (2006). Saturated fatty acids promote endoplasmic reticulum stress and liver injury in rats with hepatic steatosis. Endocrinology 147, 943-951. doi: 10.1210/en.2005-0570

Yasuda-Yamahara, M., Kume, S., Yamahara, K., Nakazawa, J., Chin-Kanasaki, M., Araki, H., et al. (2015). Lamp-2 deficiency prevents high-fat diet-induced obese diabetes via enhancing energy expenditure. Biochem. Biophys. Res. Commun. 465, 249-255. doi: 10.1016/j.bbrc.2015.08.010

Yuan, F., Teng, X., Guo, Z., Zhou, J. J., Zhang, Y., and Wang, S. (2015). Chronic intermittent hypobaric hypoxia ameliorates endoplasmic reticulum stress mediated liver damage induced by fructose in rats. Life Sci. 121, 40-45. doi: 10.1016/j.lfs.2014.11.019

Zhang, C., Chen, X., Zhu, R. M., Zhang, Y., Yu, T., Wang, H., et al. (2012). Endoplasmic reticulum stress is involved in hepatic SREBP-1c activation and lipid accumulation in fructose-fed mice. Toxicol. Lett. 212, 229-240. doi: 10. 1016/j.toxlet.2012.06.002

Zhang, X. Q., Xu, C. F., Yu, C. H., Chen, W. X., and Li, Y. M. (2014). Role of endoplasmic reticulum stress in the pathogenesis of nonalcoholic fatty liver disease. World J. Gastroenterol. 20, 1768-1776. doi: 10.3748/wjg.v20.i7.1768

Zhao, Q., Hu, X., Shao, L., Wu, G., Du, J., and Xia, J. (2014). LipoxinA4 attenuates myocardial ischemia reperfusion injury via a mechanism related to downregulation of GRP-78 and caspase-12 in rats. Heart Vessels 29, 667-678. doi: 10.1007/s00380-013-0418-y

Zhou, J. J., Wei, Y., Zhang, L., Zhang, J., Guo, L. Y., Gao, C., et al. (2013). Chronic intermittent hypobaric hypoxia prevents cardiac dysfunction through enhancing antioxidation in fructose-fed rats. Can. J. Physiol. Pharmacol. 91, 332-337. doi: 10.1139/cjpp-2012-0059

Conflict of Interest: The authors declare that the research was conducted in the absence of any commercial or financial relationships that could be construed as a potential conflict of interest.

Copyright (c) $2020 \mathrm{Cui}, \mathrm{Hu}, \mathrm{Guo}$, Sun and Shi. This is an open-access article distributed under the terms of the Creative Commons Attribution License (CC BY). The use, distribution or reproduction in other forums is permitted, provided the original author(s) and the copyright owner(s) are credited and that the original publication in this journal is cited, in accordance with accepted academic practice. No use, distribution or reproduction is permitted which does not comply with these terms. 